\title{
Politique pour une gestion des " déchets " flottants
}

\author{
Policy for a floating “ waste " management \\ par Franck Darthou \\ Mission Technique Commune \\ EDF / Agence de l'Eau Adour-Garonne
}

Today, the problem of Floating " Waste " imposes itself at all levels : deposits along the banks of rivers, on lakes and on beaches of the coast, threat to stability of some works, difficulties in exploiting hydroelectric power are some of the concerns to the administrators and users of our rivers.

Despite themselves, dams play a part in gathering floating " waste ". Today, except in particular cases, no regulations exist on the owners of these dams to take the floating " waste " out the river.

The Master Plan (" Schéma Directeur d'Aménagement et de Gestion des Eaux ") of the Adour-Garonne Basin is recommending, however, the management of floating " waste" on a basin scale.

The aim, on the one hand, is to organize the relevant conditions for the recovery of the floating " waste ", and, on the other hand, to create a synergy between all the parties for their processing and their recycling.

\section{LA PROBLÉMATIQUE GÉNÉRALE DES “ DÉCHETS " FLOTTANTS}

\section{- 1.1 Le contexte général}

Chacun connaît la fonction première des rivières à drainer les eaux des bassins versants qu'elles traversent. La nature de ces derniers, l'occupation des sols et la traversée de zones urbanisées, industrialisées ou touristiques conferrent, en outre, aux cours d'eau la capacité de collecter quantités de résidus flottants. Ainsi, troncs, broussailles, feuilles, planches, plastiques, bidons,... viennent encombrer le lit des cours d'eau et des retenues, peuvent perturber l'écoulement des eaux, être susceptibles de dégrader la qualité des eaux ou déterminer un impact direct sur l'environnement paysager :

- des rivières par stagnation le long des berges et des zones d'eaux mortes :

- des retenues par stagnation au droit des barrages et des prises d'eau :

- du littoral par transfert des "déchets" au droit des estuaires.

Ce phénomène de " drainage " des " déchets " flottants peut, en outre, être considérablement accentué par :

- l'entretien déficitaire du lit et des berges des cours d'eau par les propriétaires riverains,

- l'image de nos cours d'eau considérés encore trop souvent comme des " dépotoirs " plutôt que comme un élément essentiel de notre patrimoine.

En outre, les " déchets " flottants, en s'accumulant au droit des grilles de prises d'eau, peuvent constituer une gêne pour l'exploitation des ouvrages hydroélectriques.
Les barrages sont de simples concentrateurs de "déchets " et aujourd hui, la réglementation autorise les exploitants à les rejeter en aval des installations par des dromes, des dégrilleurs ou tout simplement par ouverture de vannes ou de clapets de surface.

Il apparaît donc que la gestion des " déchets " flottants relève d'une problématique générale qui, pour être efficace, doit être appréhendée dans sa globalité. Elle doit mettre en œuvre un programme d'actions complémentaires intégrant l'ensemble des composantes suivantes :

- entretien des berges,

- sensibilisation et information auprès des pollueurs potentiels,

- récupération au niveau des aménagements hydrauliques,

- collecte et traitement adapté.

\subsection{Le SDAGE Adour-Garonne}

$\mathrm{Si}$ actuellement, dans la plupart des cas, aucune obligation n'est faite aux exploitants d'ouvrages hydroélectriques en matière de récupération des " déchets " flottants, leur présence ne leur étant pas imputable, l'évolution permanente de la réglementation en matière de gestion des eaux et des déchets permet d'appréhender la mouvance du sujet et l'intérêt qu'il faut lui consacrer.

Ainsi, conformément à la Loi sur l'Eau du 3 janvier 1992 et afin de fixer les principes fondamentaux de la gestion équilibrée de la ressource en eau, les Schémas Directeurs d'Aménagement et de Gestion des Eaux (SDAGE) ont été adoptés en 1996 pour chacun des six bassins hydrographiques français. 
Ces documents établissent un ensemble de dispositions avec lesquelles doivent être compatibles les programmes et les décisions administratives dans le domaine de l'eau.

Ainsi par exemple, le SDAGE Adour-Garonne recommande le développement d'une véritable politique structurelle d'entretien des berges (gestion de la ripisylve et résorption des dépôts sauvages) ainsi qu'une organisation concertée de récupération et de traitement des " déchets " flottants. Ainsi, dans sa mesure A17, il recommande que :

- - les autorisations d'exploitation des ouvrages hydrauliques prescrivent les modalités de récupération des déchets flottants,

- les autorisations en cours soient rendues compatibles avec l'alinéa ci-dessus dans un délai de 5 ans après approbation du SDAGE,

- dans le cadre de schémas définis par cours d'eau ou bassin versant :

- la nature et le volume de ces "déchets" soient pris en compte par les plans départementaux d'élimination des déchets ménagers et assimilés,

- la récupération des déchets soit faite sur chaque ouvrage, ou sur certains d'entre eux par les exploitants de ces ouvrages, - des contrats de bassins versants entre exploitants d'ouvrages, collectivités d'amont et d'aval et l'Etat, règlent les modalités de récupération, de collecte et de traitement de l'intégralité des déchets en cohérence avec les plans cités ci-dessus ".

\section{II $\square$ UN NÉCESSAIRE PREMIER ÉTAT DES CONNAISSANCES}

La gestion des " déchets " flottants au niveau des aménagements hydrauliques en général et hydroélectriques en particulier reste une préoccupation relativement récente. Son intérêt est notamment apparu avec le renouvellement de plusieurs titres administratifs et lors de l'élaboration du SDAGE, plus particulièrement sur le bassin Adour-Garonne.

C'est ainsi que, dans le cadre d'une démarche commune, l'Agence de l'Eau Adour-Garonne et EDF ont réalisé, en 1995, un premier rapport de synthèse à caractère bibliographique intitulé " Récupération et traitement des " déchets " flottants Recensement des dispositions existantes".

$\mathrm{Ce}$ document a pour objectif de fournir une vision globale de la problématique en l'abordant notamment sur les plans logistique, technique et financier.

Ainsi, sont inventoriées et décrites les techniques envisageables pour la récupération, l'élimination et la valorisation des " déchets " flottants.

Pour la récupération, on peut ainsi retenir les techniques :

- d'interception par barrage flottant ou drome,

- de récupération par bateau de type faucardeur,

- de dégrillage.

L'élimination, quant à elle, peut être envisagée par :

- une mise en décharge, qui compte tenu de la réglementation en vigueur (au ler juillet 2002 ne sont admis en décharge que les "résidus ultimes") ne peut être considérée que comme une solution transitoire,

- une incinération contrôlée.

Enfin, la valorisation, lorsqu'elle est possible à un coût acceptable et qu'elle ne pose pas de problème d'environnement, est à privilégier. Suivant le type de " déchets ", les volumes en jeu et les filières potentielles, différents types de valorisation peuvent être envisagés. Citons par exemple :

- l'incinération avec récupération de chaleur (chaufferies bois, cogénérations bois,...) qui pour les " déchets " flottants nécessite :

- un tri minimum, compte tenu de l'hétérogénéité des “ déchets" (de la présence éventuelle de métaux non broyables et non incinérables, de bouteilles de gaz entraînant des risques d'explosion,...),

- un broyage préalable des bois,

- un essorage voire un séchage afin d'améliorer le pouvoir calorifique.

— Le compostage : les " déchets " flottants végétaux peuvent contribuer, en mélange avec d'autres apports organiques, à la fabrication de compost. Cette valorisation implique notamment :

- un tri préalable performant afin de ne conserver que la partie végétale,

- un broyage spécifique afin de provoquer une meilleure altération mécanique des tissus ligneux et ainsi de favoriser l'attaque microbienne ultérieure.

D'autre part, le rapport de synthèse EDF/Agence de l'Eau recense les principales réalisations mettant en œuvre des dispositifs de récupération, de collecte et de traitement des " déchets" flottants.

Parallèlement à cette étude générique, EDF, en partenariat avec l'Agence de l'Eau Adour-Garonne, a été à l'initiative d'un projet pilote sur le barrage hydroélectrique de Luzech (46) situé sur le Lot en aval de Cahors.

Le choix de ce site a été motivé par une implantation en un point médian du bassin du Lot engendrant une accumulation importante de " déchets " flottants au droit des grilles de la prise d'eau usinière. Actuellement, ces " déchets " sont évacués vers l'aval, soit par dégrillage, soit par abaissement d'un clapet de surface.

Ce projet avait pour principal objectif d'identifier, sur un cycle annuel, les flux de " déchets" flottants au niveau de l'ouvrage.

Ainsi, la nature et les quantités de " déchets " flottants ont été établies en considérant notamment la répartition annuelle des apports et l'éventuelle corrélation avec l'hydraulicité.

Les principaux résultats de ce suivi sont présentés dans l'encadré

D'octobre 1994 à août 1995 , près de $2100 \mathrm{~m}^{3}$ de " déchets" flottants ont été évacués par chasse et dégrillage au barrage de Luzech (46) sur le Lot. Soit : - $1300 \mathrm{~m}^{3}$ de bois, $750 \mathrm{~m}^{3}$ de feuilles et fleurs, $25 \mathrm{~m}^{3}$ de " déchets" ménagers (plastiques, verre, métaux,...), les " déchets" transitant au moment des crues n'étant pas comptabilisés. (Nota : En crue, il passe plus de $\mathbf{5 0 \%}$ du volume total des " déchets" transitant annuellement au barrage).

- Les maxima sont de $600 \mathrm{~m}^{3}$ de " déchets" par mois, et de $325 \mathrm{~m}^{3}$ en un jour (début de crue).

- La quantification des erreurs d'estimation tend à multiplier les quantités brutes de l'étude par 1,7 , pour obtenir les chiffres qui pourraient servir à un dimensionnement de dispositifs de récupération, de collecte ou de traitement. 
L'ensemble du travail de collecte de données, d'identification dęs flottants et d'analyse des résultats constituera, à l'évidence une source d'informations appréciable pour la mise en place d'une récupération de "déchets " flottants sur d'autres sites du bassin.

\section{III — DES SCHÉMAS DIRECTEURS POUR UNE MEILLEURE GESTION DES “ DÉCHETS ” FLOTTANTS}

\section{1 Les objectifs}

Afin de mettre en œuvre la recommandation A17 du SDAGE Adour-Garonne, l'Agence de l'Eau a proposé l'élaboration de schémas directeurs de gestion des "déchets " flottants par grand bassin versant. L'objectif est d'établir un plan d'actions global et cohérent de la gestion de ces " déchets " afin d'éviter une récupération systématique au niveau de chaque obstacle qui les retient (notamment les ouvrages hydroélectriques) ce qui ne constitue pas la solution la plus judicieuse et la plus efficace, d'autant qu'elle pose systématiquement le problème de l'élimination.

Ces schémas directeurs s'articulent autour de deux axes complémentaires :

- la récupération, nécessitant une organisation concertée des exploitants d'ouvrages hydrauliques d'amont en aval du cours d'eau ;

- la collecte et le traitement, nécessitant l'implication des professionnels et des collectivités compétentes d'amont en aval de la chaîne du " déchet ".

Ces schémas doivent constituer le cadre opérationnel d'une politique globale et cohérente partagée par l'ensemble des partenaires concernés. Ils doivent ainsi :

— déterminer la nature des " déchets " flottants à récupérer ;

- déterminer les sites les plus adaptés d'une part pour la récupération, d'autre part pour la collecte et le traitement. Ainsi, par exemple, sont proposés des sites dits " stratégiques " de récupération dont le choix se justifie principalement par rapport :

- aux caractéristiques du site afin de déterminer la faisabilité technique d'une récupération efficace ainsi que les possibilités locales de collecte et de traitement,

- à la position du site sur le bassin versant afin de replacer l'aménagement dans son environnement général en appréhendant les impacts induits à l'aval, le potentiel d'apport de " déchets " flottants eu égard à la situation relative des sites par rapport aux confluences, aux agglomérations, à l'occupation des sols, à la morphodynamique du cours d'eau...

- s'inscrire dans la démarche engagée au niveau départemental qui doit conduire à la mise en œuvre de Plans départementaux d'élimination des " déchets " ménagers et, éventuellement, dans les démarches relatives aux SAGE (Schémas d'Aménagement et de Gestion des Eaux) ou aux contrats de rivière ;

- élaborer une politique pluriannuelle des actions à mener ;

- estimer les moyens à dégager en termes d'investissement, d'exploitation et d'organisation ;

- déterminer une planification des dépenses à engager ;

- définir les partenaires financiers et mettre en place les plans de financements correspondants.

\section{- 3.2 Les principes de mise en œuvre de la démarche}

La collecte et le traitement des " déchets " flottants reste plus un problème d'ordre logistique et économique qu'un défi technique.

L'enjeu fondamental est donc de créer une dynamique autour de cette problématique en recherchant l'implication à différents niveaux de l'ensemble des partenaires concernés.

Ainsi pour le bassin Adour-Garonne, l'Agence de l'Eau a suscité la concertation à différents niveaux :

- Tout d'abord à l'échelle de l'ensemble du bassin AdourGaronne, elle a organisé le rapprochement de l'ensemble des interlocuteurs de bassin (DRIRE, DIREN, ADEME, principaux producteurs hydroélectriques,...) afin de définir la stratégie globale dont les grandes lignes sont :

a/ l'élaboration de schémas directeurs de gestion des " déchets " flottants à l'échelle de chacun des sous-bassins hydrographiques suivants : Charente, Dordogne, Lot, Tarn, Agout, Garonne, Ariège, Neste, Adour. Ce découpage géographique permet notamment de :

- prendre en compte la spécificité des grandes unités hydrauliques ou hydrogéologiques ;

- identifier au mieux les problèmes ;

- assurer la cohérence technique des projets ;

- identifier au mieux les interlocuteurs et les responsabilités de chacun ;

- favoriser la concertation et faciliter les négociations ultérieures.

b/ l'information de la démarche auprès de l'ensemble des MISE (DDE/DDAF), DRIRE et DIREN du bassin AdourGaronne afin de les associer dès le départ à la réflexion et qu'ils puissent, le cas échéant, en tenir compte dans l'exercice de leurs missions réglementaires notamment dans le cadre des renouvellements de titre administratif d'aménagements hydroélectriques.

c/ l'appropriation par un maître d'ouvrage ayant une légitimité à l'échelle du sous-bassin considéré. A cet effet, l'implication directe des collectivités locales (Conseils Généraux, Ententes ou Institutions Interdépartementales là où elles existent) a été recherchée pour piloter les projets,

- Localement, l'ensemble des partenaires sont associés à la démarche dans le cadre de comités de pilotage qui contribuent à :

- l'établissement des cahiers des charges pour l'élaboration des schémas directeurs en prenant en compte les spécificités du bassin versant considéré ;

- la validation des différentes propositions émises ;

- la mise en œuvre effective des orientations retenues.

\section{- 3.3 Un point sur l'état d'avancement de la démarche en Adour-Garonne}

Cette politique de gestion globale et cohérente des " déchets " flottants à l'échelle d'un bassin versant reste aujourd'hui une initiative prise principalement au niveau du bassin Adour-Garonne.

Sur ce bassin, à l'automne 1998, l'élaboration des schémas directeurs était d'ores et déjà engagée sur plusieurs unités hydrographiques :

— sur le bassin de l'Adour où la maîtrise d'ouvrage des études est assurée par l'Institution Interdépartementale pour l'Aménagement Hydraulique du Bassin de l'Adour, cette démarche intègre, outre les aspects fluviaux : 


\section{Eau : ressources, aménagements, envifonnement}

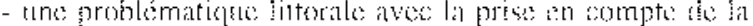

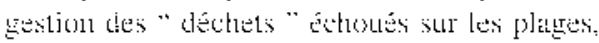

- une problematjake transfrontaliote aves un perimetre

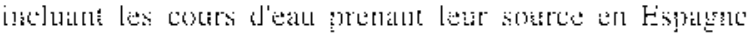

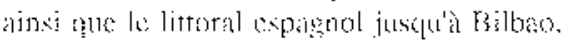

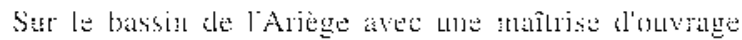

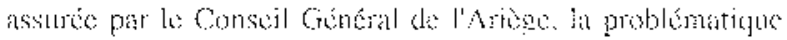

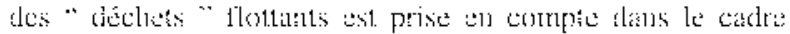
d'une crude plus globals de ec balsisu.

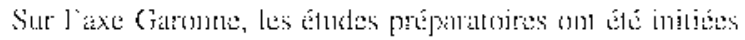

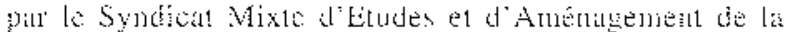

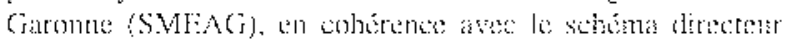

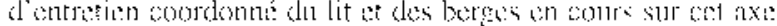

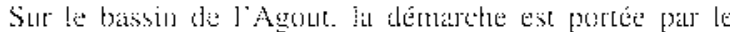
Syndicat Mixes Therestomer.

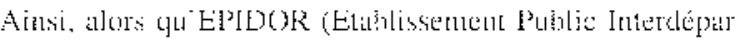

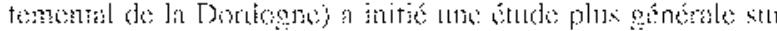

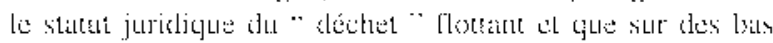

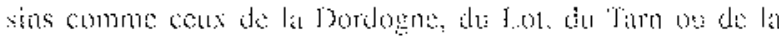

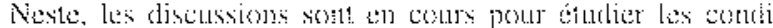

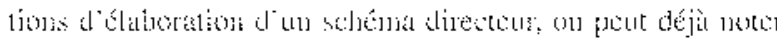

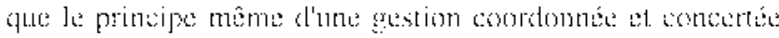

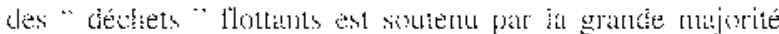

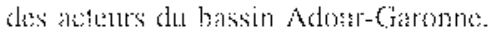

